ÉDITORIAL / EDITORIAL

\title{
COVID-19 : les sociétés savantes doivent se réinventer dans le monde d'après
}

\section{COVID-19: Learned societies must reinvent themselves in the post world}

Hervé Maisonneuve ${ }^{1 \star}$ • Didier Dreyfuss ${ }^{2} \cdot$ Michel Dubois $^{3}$

Reçu le 2 juin 2020 ; accepté le 10 juillet 2020.

(c) SRLF 2020.

"N'oublions pas que le progrès est un objectif optionnel, et non un engagement inconditionnel, et que son rythme en particulier, aussi compulsif soit-il, n'a rien de sacré. Souvenons-nous aussi qu'une progression plus lente dans la conquête de la maladie ne menacerait pas la société... mais que la société serait en effet menacée par l'érosion de ces valeurs morales dont la perte, peut-être causée par une poursuite trop acharnée du progrès scientifique, enlèverait toute valeur à ses triomphes les plus éclatants. "

Hans Jonas, philosophe et éthicien, auteur du « Principe de Responsabilité » [1]

\section{Introduction}

Lors de la pandémie due au SARS-CoV-2, les médias ont atteint l'un de leurs objectifs : faire de l'audience ! Les chaînes d'information en continu, soucieuses d'alimenter le moulin à paroles dont elles dépendent, n'ont souvent pas su distinguer l'expert du pseudo-expert n'ayant rien à dire. Les réseaux sociaux - en particulier Facebook et Twitter - ont nourri la confusion entre information scientifique et infox, lorsqu'il ne s'agissait pas simplement de diffuser les visions complotistes générées par l'épidémie virale. Cette circulation accélérée et dérégulée de l'information par temps de pandémie a eu des effets collatéraux délétères.

Gérer l'incertitude qu'un véritable expert doit rendre perceptible est un exercice difficile tant pour les journalistes que pour un public en quête de certitudes. D'où parfois le recours à des pseudo-experts, venus de nulle part, et reconvertis pour l'occasion en spécialiste de réanimation, de virologie, d'infectiologie, etc. Les débats autour de la COVID-19 ont montré que la notion de données acquises de la science était ignorée par un grand nombre d'intervenants. La chloroquine a été traitée en oubliant totalement les leçons du benfluorex (Médiator). Les journalistes scientifiques étant peu nombreux, des journalistes formés dans l'urgence ont animé des débats en confondant données probantes et opinions. Une partie de l'opinion, pressée d'obtenir des réponses immédiates, semble sensible aux messages de ceux qui prétendent traiter des malades là où les autres ne feraient au mieux que traiter des dossiers, de ceux qui considèrent que le temps accéléré de la pandémie est incompatible avec le temps forcément plus long de la recherche, etc.

\section{Les sociétés savantes n'ont pas été suffisamment présentes dans ces débats}

Permettez d'être naïf et de rêver : les sociétés savantes, dont la responsabilité est aussi de maîtriser les connaissances propres à leur domaine d'expertise, pourraient être consultées par les médias pour dissocier l'expert du

\footnotetext{
*Dr Hervé Maisonneuve

Rédacteur de https://www.redactionmedicale.fr , 30 avenue Faidherbe, 75011 Paris, France

Comité scientifique, IRAFPA (Institute of Research and Action on Fraud and Plagiarism in Academia), Genève, Suisse

$\nabla$ hervemaisonneuve@gmail.com

La liste complète des auteurs est disponible à la fin de l'article.
} 
pseudo-expert. Pourquoi, cela n'a-t-il pas été le cas ? Les invitations faites par les rédactions sont probablement basées sur une multitude de facteurs : savoir parler, être à proximité (ou par visioconférence), disponibilité, connivences entre journalistes et un cercle d'influenceurs, théâtralité de l'orateur, personnalité publique, comme des hommes politiques, etc.

Même s'il existe plusieurs façons de remplir le rôle d'expert [2], ne parler que dans son domaine de compétence est sans doute une caractéristique importante. Quand un politique prescrit un médicament à la télévision, ce n'est pas son rôle. Quand un journal quotidien fait un sondage pour savoir si un médicament est efficace, il entretient un populisme médical dangereux. Quand des politiques et médecins font signer des pétitions pour faire prescrire un médicament sans s'assurer des risques, ce n'est pas leur rôle. L'efficacité d'un médicament ne peut pas être établie par sondage, par opinions, par croyances dans un pays où notre démocratie a délégué ce rôle à une Agence du médicament. Cette Agence aurait pu être proactive, soit en demandant aux fabricants d'avoir accès aux données probantes justifiant la prescription, soit en auditant sur site des essais cliniques en cours et peut-être également en opposant une résistance plus ferme aux exigences médiatisées. De même, la justification par des experts scientifiques, à la compétence par ailleurs indiscutable, de décisions politiques motivées par des raisons en fait logistiques a traduit une confusion des rôles qui n'était pas souhaitable. On l'a constaté à propos de l'utilité des masques.

Les sociétés savantes ont un rôle à jouer pour conseiller ces Agences, les administrations. II leur revient par temps de crise d'investir l'espace public et d'être présentes sur les plateaux de télévision, avec leur savoir, leur image, voire leur logo.

\section{Les revues scientifiques ont été dépassées par les préprints et les réseaux sociaux}

Des générations entières de chercheurs ont respecté des principes de contrôle de la communication scientifique édictés par les sociétés savantes et leurs revues. Ces dernières s'engageaient à ne pas considérer des manuscrits dont les résultats avaient été préalablement communiqués au public. Cette règle proposée en 1969 par Franz Ingelfinger, rédacteur en chef du New England Journal of Medicine à l'époque, puis revisitée en 1991 lors de l'apparition du SIDA, était admise [3, 4]. Le développement des technologies numériques couplées à la diffusion croissante des thématiques de l'ouverture et de la participation ont progressivement fait oublier cette règle. L'arrivée des préprints en médecine vers le milieu de 2019, puis leur explosion avec la pandémie (sur medRxiv, 6005 préprints COVID-19 ont été déposés entre le 1 janvier et le 14 août 2020) semblent fragiliser le modèle traditionnel d'évaluation et de diffusion des données scientifiques par les revues (voir sur medrxiv.org). Les discussions scientifiques se sont déportées vers les réseaux sociaux (Twitter, Facebook, Linkedln, PubPeer, YouTube, etc.), avec une accessibilité croissante pour le citoyen informé comme le public naïf... Que doivent faire les sociétés savantes?

\section{Quelles sont les missions des sociétés savantes ?}

L'un de nous trois avait travaillé sur une définition des sociétés savantes entre 1997 et 2004 quand les structures de l'évaluation médicale se développaient en France, à savoir l'Agence Nationale pour le Développement de l'Évaluation Médicale, puis l'Agence Nationale d'Évaluation et d'Accréditation en Santé. Le fonctionnement des sociétés savantes était basé sur le volontariat de professionnels éloignés, communiquant par courrier, téléphone puis courriel [5]. Le rythme des réunions ne permettait pas toujours de répondre rapidement à des demandes de l'administration. Les sociétés savantes avaient un désir de reconnaissance, un fonctionnement interne variable et un manque de moyens en regard de leurs ambitions. La définition proposée mériterait actualisation en 2020 [6, 7]. Cette définition était : «Groupe organisé, dans un champ disciplinaire donné, dont les adhérents ont pour objectif de rendre compte de leurs travaux, d'améliorer la connaissance dans leur domaine, d'assurer la formation et la recherche, de diffuser les résultats de leurs activités, de soutenir et promouvoir leur discipline ». Cette définition a été construite à partir des réponses de 129 sociétés savantes sur les 225 sollicitées (57\%).

\section{Une société savante doit s'adresser au citoyen autant qu'à ses membres}

La plupart des sociétés savantes ont réagi à la pandémie en s'exprimant par le biais de leur site internet. Elles ont mis en ligne des recommandations, des liens vers des sources qualifiées, des analyses de littérature, des communiqués, des vidéos pour apporter de l'information. Cette information libre d'accès était très spécialisée et surtout dirigée vers les membres de la société. N'est-il pas naturel que les sociétés savantes ne s'adressent en priorité qu'à leurs membres ? Sauf erreur de notre part, seule La Société Française de Pharmacologie et de Thérapeutique (SFPT), en partenariat avec le Réseau Français des Centres Régionaux de Pharmacovigilance, le Collège National des Enseignants de Thérapeutique le Collège National de Pharmacologie Médicale, l'Association Française des Centres d'Addictovigilance, I'Association Nationale des Enseignants de Pharmacie Clinique, et srlf 
en lien avec l'ANSM et le Ministère des Solidarités et de la Santé (DGS), a mis en ligne des réponses à 164 questions (28 juin 2020) (voir ces questions-réponses sur le site de la SFPT : https://sfpt-fr.org/covid19). La Société de Réanimation de Langue Française (srlf.org) et la Société Française d'Anesthésie et de Réanimation (sfar.org) ont été très actives avec des recommandations pour la prise en charge des malades COVID-19 graves, une veille bibliographique et des vidéos pour leurs membres. De nombreuses sociétés savantes ont produit des informations, des avis scientifiques et/ou des veilles documentaires pour leurs membres avec un accès libre : Société de Pathologie Infectieuse de Langue Française (infectiologie.com), Société Française de Médecine d'Urgence (sfmu.org), Société de Pneumologie de Langue Française (splf.fr), Société Française de Cardiologie (sfcardio.fr), Société Française de Dermatologie (sfdermato.org), Société Française de Santé Publique (sfsp.fr), Société Nationale de Médecine Interne (snfmi.org), Collège National des Généralistes Enseignants (cnge.fr). Des sociétés savantes ont produit des informations réservées à leurs membres, accessibles avec des codes, par exemple la Société Française de Chirurgie Thoracique et Cardio-vasculaire (sfctcv.org).

L'Académie Nationale de Médecine, habituellement réservée, s'est énervée avec un communiqué de presse du 8 mai 2020 [8] : "La vérité scientifique ne se décrète pas à l'applaudimètre. Elle n'émerge pas du discours politique, ni des pétitions, ni des réseaux sociaux. En science, ce n'est ni le poids majoritaire ni l'argument d'autorité qui font foi ». Ce communiqué s'adresse au public autant qu'aux chercheurs. Peut-on assimiler l'Académie de Médecine à une société savante ? Est-ce que l'Académie serait une structure fédératrice des sociétés savantes ? A-t-on besoin d'une structure fédératrice des sociétés savantes?

Les sociétés savantes ont été confrontées à l'annulation d'évènements, et ont utilisé des moyens électroniques de communication. Certaines ont organisé des congrès virtuels. Les sociétés vont être questionnées par les plus jeunes : "Le congrès virtuel était-il réussi avec de nouveaux participants, avec parfois plus de participants, pour des coûts raisonnables en diminuant l'empreinte carbone ? Qu'allez-vous faire après la COVID-19? ». Ce n'est qu'une des interrogations des sociétés savantes pour aborder le monde d'après... les autres étant le modèle économique, la gestion des connaissances, voire l'utilité et l'impact des congrès [9]. Faut-il revoir les missions des sociétés savantes pour s'assurer d'une présence nécessaire dans les débats publics?

II paraît urgent d'entreprendre un véritable état des lieux pour reconsidérer la définition proposée en 2004 [6], pour cartographier les canaux de communication des sociétés savantes, en particulier leur présence sur les réseaux sociaux. La confiance du public dans la science nécessite de repenser le rôle comme la valorisation de ces acteurs légitimes de la science que sont les sociétés savantes.

\section{Remerciements}

Nous remercions Anne Boutet et Sandrine Chauve.

\section{Conflits d'intérêts}

Hervé Maisonneuve, Didier Dreyfuss et Michel Dubois déclarent ne pas avoir de conflit d'intérêts.

\section{Affiliations}

${ }^{1} 30$ rue Faidherbe, 75011 Paris

${ }^{2}$ Université de Paris

Hôpital Louis Mourier, Médecine Intensive-Réanimation

Colombes (Assistance Publique-Hôpitaux de Paris)

${ }^{3}$ CNRS, Groupe d'Étude des Méthodes de l'Analyse Sociologique

de la Sorbonne (GEMASS)

59-61 CNRS Site Pouchet, 75017 Paris

\section{Références}

1. Jonas H. Philosophical reflections on experimenting with human subjects. Dadedalus, Journal of the American Society of Arts and Sciences 1969;98:219-47.

2. Pielke J Roger A (2007) The Honest Broker: Making Sense of Science in Policy and Politics. Cambridge University Press, Cambridge

3. (1969) Definition of Sole Contribution. New England Journal of Medicine 281:676-677. DOI : 10.1056/NEJM196909182811208

4. Angell M, Kassirer JP (1991) The Ingelfinger Rule Revisited. New England Journal of Medicine 325:1371-1373. DOI : 10.1056/NEJM199111073251910

5. Fourquet F, Maisonneuve H, Steudler F, et al (1997) L'organisation des sociétés médicales en France. Presse Med 26:715-718

6. Moreau N, Maisonneuve H, Steudler F, Durocher A (2004) Pour une meilleure compréhension des sociétés savantes médicales françaises. Cah Sociol Demogr Med 44:365-389

7. Maisonneuve H, Moreau N, Steudler F, et al (2004) Typologie des sociétés savantes médicales françaises. Presse Med 33:784-790. DOI : 10.1016/s0755-4982(04)98744-1

8. Académie nationale de médecine. Recherche clinique et Covid-19: la science n'est pas une option. Communiqué du 8 mai 2020 - consulté le 14/08/2020 http://www.academie-medecine.fr/communique-delacademie-nationale-de-medecine-recherche-clinique-et-covid-19-lascience-nest-pas-une-option/

9. Ioannidis JPA (2012) Are medical conferences useful? And for whom? JAMA 307:1257-1258. DOI : 10.1001/jama.2012.360 
